\title{
Combining visibilities from the giant meterwave radio telescope and the Nancay radio heliograph
}

\author{
High dynamic range snapshot images of the solar corona at $327 \mathrm{MHz}$
}

\author{
C. Mercier ${ }^{1}$, P. Subramanian ${ }^{2}$, A. Kerdraon ${ }^{1}$, M. Pick ${ }^{1}$, S. Ananthakrishnan ${ }^{3}$, and P. Janardhan ${ }^{4}$ \\ 1 Observatoire de Paris, LESIA, 5 place Janssen, 92190 Meudon, France \\ e-mail: claude.mercier@obspm.fr \\ 2 Inter-University Centre for Astronomy and Astrophysics, PO Bag 4, Ganeshkhind, Pune-411007, India \\ 3 NCRA - TIFR, PO Bag 3, Ganeshkhind, Pune University Campus, Pune-411007, India \\ ${ }^{4}$ Physical Research Laboratory, Astronomy \& Astrophysics Division, Navrangpura, Ahmedabad-380009, India
}

Received 13 June 2005 / accepted 1 October 2005

\section{ABSTRACT}

We report first results from an ongoing program of combining visibilities from the Giant Meterwave Radio Telescope (GMRT) and the Nancay Radio Heliograph $(N R H)$ to produce composite snapshot images of the sun at meter wavelengths. We describe the data processing, including a specific multi-scale CLEAN algorithm. We present results of a) simulations for two models of the sun at $327 \mathrm{MHz}$, with differing complexity b) observations of a complex noise storm on the sun at $327 \mathrm{MHz}$ on Aug. 27, 2002. Our results illustrate the capacity of this method to produce high dynamic range snapshot images when the solar corona has structures with scales ranging from the image resolution of $49^{\prime \prime}$ to the size of the whole sun.

We emphasize that snapshot images of a complex object such as the sun, obtained by combining data from both instruments, are far better than images from either instrument alone, because their $u v$-coverages are very complementary.

Key words. Sun: radio radiation - techniques: interferometric - techniques: image processing

\section{Introduction}

We have an ongoing program of combining data from the GMRT and NRH in order to make composite meter wavelength images of the sun. Our aim is to combine the short baseline data from the $N R H$ with long baseline data from the GMRT in order to obtain meter wavelength images of unprecedented resolution and fidelity. We present here the first results from our campaign.

Imaging complex, rapidly varying radio sources in the solar corona is problematic because one needs to obtain images spanning durations as short as a few seconds. The $u v$-coverage for short duration exposures is usually quite poor, and this affects the ability to image complex sources reliably. Furthermore, deconvolution procedures like the commonly used CLEAN often perform poorly on sources that exhibit structures on a variety of scales (Wakker \& Schwarz 1991). The problem of poor $u v$-coverage can be alleviated by synthesis imaging over time durations of a few hours, but this technique cannot be used for studying phenomena that vary rapidly in time. There are several instances in solar physics where high dynamic range, high resolution snapshot images are essential. For instance, studies of extended thermal emission in the corona, coronal mass ejections (CMEs) that frequently occur together with compact, bright bursts and flare continua require imaging with a large field of view, high spatial resolution and dynamic range. High resolution observations can establish a firm limit on the angular broadening of sources in the solar corona due to turbulence, which determines the apparent size of radio bursts (e.g. Bastian 1994).

\subsection{Dynamic range}

Before proceeding further, we pause to define two kinds of dynamic ranges we will refer to below. The rms dynamic range is the definition usually employed by the community. It is the ratio of the peak brightness of the image to the rms brightness of a representative empty region near the area where the peak brightness occurs. This definition is relevant when artefacts on the final images look like random noise, which is often the case for images produced via aperture synthesis. However, we deal 
here with snapshot images, for which the visibilities are rather sparsely sampled, especially for large baselines. The artefacts on such snapshot images are localized and structured, and often look like spurious compact sources. We will use another definition of dynamic range, which we call the "max" dynamic range, which we think is more relevant to the snapshot images we present. The max dynamic range is defined as num/denom, where num is the peak brightness of the image. The quantity denom is the maximum deviation from the mean of an empty region around the area where the peak brightness occurs. We will quote the rms dynamic range for the sake of comparison with published results. The max dynamic range is typically an order of magnitude lower than the rms dynamic range for our results.

\subsection{Meter wavelength observations of the solar corona: a brief overview}

Meter wavelength observations of the solar corona to date have concentrated either on having a large field of view with dense short baseline coverage (e.g., observations using the $N R H$ ) or on achieving high spatial resolution at the expense of a sparse short baseline coverage (e.g., extended array observations with the Very Large Array (VLA)). In what follows, we attempt to give a short overview of meter wavelength imaging observations of the solar corona. This will help to place the observations reported here in context.

There have been several attempts at detecting small scale structures at meter wavelengths in the solar corona. Kerdraon (1979) used one-dimensional (1D) scans at a time cadence of $0.02 \mathrm{~s}$ and a spatial resolution of $1.2^{\prime}$ with the $N R H$ at $169 \mathrm{MHz}$. The 1D scans were obtained by a simple Fourier transform $(F T)$ of the observed complex visibilities $(C V \mathrm{~s})$. The field of view was around 1 degree, and the artefacts on the $1 \mathrm{D}$ scans were due to sidelobes of the response function. The max dynamic ranges of these 1D scans were 5-10. Kerdraon concluded that type 1 bursts at $169 \mathrm{MHz}$ range in size from $0.7^{\prime}$ to $3^{\prime}$.

Zlobec et al. (1992) have used the VLA in its A configuration with a resolution of $4^{\prime \prime}$ at $327 \mathrm{MHz}$ to search for small scale structures in snapshot maps with a time cadence of $1.6 \mathrm{~s}$. They find that the smallest type I source sizes they can observe are around $30^{\prime \prime}$. The largest structure that can possibly be imaged with the VLA A-array is around 110". However, owing to the shortage of short baseline spacings, Zlobec et al. (1992) mention that they cannot be confident about large scale structures. In some cases, they cannot be confident about the positions of the small structures. Furthermore, the rms dynamic range of the images they obtain is around 10, at best.

Some other instances of snapshot imaging of noise storm sources in the solar corona at meter wavelengths are Willson et al. (1997) (VLA C configuration, resolution of 40" at $327 \mathrm{MHz}$, time cadence $3.3 \mathrm{~s}$ ), Willson et al. (1998) (VLA A configuration, resolution $30^{\prime \prime}$ at $75 \mathrm{MHz}, 5^{\prime \prime}$ at $327 \mathrm{MHz}$, time cadence $10 \mathrm{~s}$, source size $1.5^{\prime}$ at $327 \mathrm{MHz}, 2.5 \times 5^{\prime}$ at $75 \mathrm{MHz}$ ), Willson (2000) (VLA D configuration, time cadence $3.3 \mathrm{~s}$, resolution $180^{\prime \prime}$ at $327 \mathrm{MHz}$ ).
There have also been several instances of synthesis imaging of noise storm emitting regions at meter wavelengths. Habbal et al. (1996) have used the VLA in its B configuration to observe noise storm sources with synthesis durations ranging from around $100 \mathrm{~min}$ to $3.5 \mathrm{~h}$. Their resolution was around 94" at $327 \mathrm{MHz}$. Earlier, Habbal et al. (1989) had used the VLA in its $\mathrm{C}$ configuration to image a noise storm source at $327 \mathrm{MHz}$ with a synthesis duration of around $4 \mathrm{~h}$. They were able to image structures ranging from around $80^{\prime \prime}$ to $240^{\prime \prime}$ with a resolution of $57^{\prime \prime} \times 47^{\prime \prime}$ at $327 \mathrm{MHz}$. Willson et al. (1997) have used data from the VLA C array to obtain $4 \mathrm{~h}$ synthesis images of a faint transequatorial arch measuring $600^{\prime \prime}$ at $327 \mathrm{MHz}$. In a multiwavelength study aimed at high dynamic range imaging, White et al. (1992) have used the VLA D configuration to obtain $4 \mathrm{~h}$ synthesis images of active region plages measuring around $600^{\prime \prime}$ with a resolution of around $200^{\prime \prime}$ at $327 \mathrm{MHz}$. The rms dynamic range of their $327 \mathrm{MHz}$ images was between 60 and 90. In a similar study at $1420 \mathrm{MHz}$, Gopalswamy et al. (1991) used the VLA D configuration to obtain 6-7 h synthesis images of active region plages ranging from $50^{\prime \prime}$ to $200^{\prime \prime}$ with a resolution of $50^{\prime \prime}$. The rms dynamic range of their images varies from 170 to 346 .

\subsection{Present study}

In this paper, we present composite snapshot images by combining visibilities from the GMRT and the $N R H$ for:

- simulations with two models of the radio sun at $327 \mathrm{MHz}$. The first model is relatively simple and similar in many aspects to the real case reported in this paper. The second model is more complex, and we use this in order to investigate the limits of what we can hope for with snapshot imaging;

- observations of complex and evolving noise storm emitting regions in the solar corona at $327 \mathrm{MHz}$ on Aug. 27, 2002. These observations use an integration time of $17 \mathrm{~s}$. They illustrate the capabilities of the composite instrument with real data, but since solar bursts vary over time scales that are much shorter than $17 \mathrm{~s}$, they are of limited use in arriving at astrophysical results.

This procedure complements the dense short baseline coverage of the $N R H$ with the long baseline coverage of the GMRT. We obtain snapshot maps of structures with sizes ranging between the resolution $\left(49^{\prime \prime}\right)$ and the size of the whole sun with unprecedented (rms) dynamic ranges of 250-420.

\section{The instruments}

The Nancay RadioHeliograph $(N R H)$ and the Giant Meterwave Radio Telescope (GMRT) both operate at meter wavelengths, and we currently have common observations of the solar corona at 236 and $327 \mathrm{MHz}$. The two instruments have up to $4 \mathrm{~h}$ of common observing time each day. The following subsections give brief overviews of the two instruments. 


\subsection{The Nancay radio heliograph $(\mathrm{NRH})$}

The $N R H$ (Kerdraon \& Delouis 1997) is located about $200 \mathrm{~km}$ south of Paris (latitude $47^{\circ} 23^{\prime} \mathrm{N}$, longitude $2^{\circ} 12^{\prime} \mathrm{E}$, altitude $150 \mathrm{~m}$ ). It consists of 44 antennas of size ranging from 2-10 m, spread over two arms (EW and NS) with respective lengths of $3200 \mathrm{~m}$ and $2440 \mathrm{~m}$. The EW and NS baselines $d_{\mathrm{EW}}$ and $d_{\mathrm{NS}}$ are from 0 to $3200 \mathrm{~m}$ (resp $2440 \mathrm{~m}$ ) by steps $100 \mathrm{~m}$ (resp $54 \mathrm{~m}$ ). After January 2003 the number of antennas were raised to 48 and the EW minimum baseline is reduced to $50 \mathrm{~m}$. The $N R H$ observes the sun $7 \mathrm{~h}$ per day at up to 10 frequencies between 150 and $450 \mathrm{MHz}$. The standard observing mode consists of Stokes $I$ and $V$ images at 164, 236, 327, 410 and $432 \mathrm{MHz}$ each $150 \mathrm{~ms}$. The resolution of two dimensional (2D) images and the field of view depend on the frequency and the season. For the observation reported here they are $\sim 4.8^{\prime} \times 3.0^{\prime}$ and $\sim 40^{\prime} \times 70^{\prime}$ respectively.

\subsection{The giant meterwave radio telescope (GMRT)}

The GMRT is located about $80 \mathrm{~km}$ north of the city of Pune in Maharashtra, western India (latitude $19^{\circ} 06^{\prime} \mathrm{N}$, longitude $74^{\circ} 03^{\prime} \mathrm{E}$, altitude $650 \mathrm{~m}$ ). It consists of thirty $45 \mathrm{~m}$ diameter antennas spread over $25 \mathrm{~km}$. Half of these are in a compact, randomly distributed array of about 1 square $\mathrm{km}$ and the rest are spread out in an approximate "Y" configuration. The GMRT currently operates in the frequency bands around 235, 325, 610 and $1000-1450 \mathrm{MHz}$, with bandwidths ranging from 16 to $32 \mathrm{MHz}$. Of these, the 235 and $327 \mathrm{MHz}$ frequencies are most commonly used for solar observations. The shortest baseline is $100 \mathrm{~m}$ and the longest one is $26 \mathrm{~km}$. Data is usually acquired at a time cadence of $16 \mathrm{~s}$, but it is presently possible to acquire data at intervals as short as $2 \mathrm{~s}$. The automatic level control circuitry in the GMRT data processing electronics has a response time of around $1 \mathrm{~s}$. This limits the shortest integration time to be $\approx 2 \mathrm{~s}$, which is still substantially longer than what can be achieved with the $N R H$. Solar observations are typically carried out from around 03:30 UT to 12:30 UT. During such observations, the sun is typically observed for around $15-20 \mathrm{~min}$, after which a nearby phase calibrator is observed for around $10 \mathrm{~min}$. This cycle is then repeated. During a particular cycle, the antennas track the sun at a linear rate in RA and Dec. At the beginning of the next cycle, the antennas are repositioned to the RA and Dec of the sun corresponding to the time when the cycle begins, and they then track the sun as before. Further details about the GMRT can be found at the URL http://www.gmrt.ncra.tifr.res.in and in Ananthakrishnan \& Rao (2002), Swarup et al. (1991).

\subsection{The composite instrument}

It is useful to discuss at this point some properties of the composite instrument, for a better understanding of the subsequent data processing. Figure 1 shows the $u v$ coverage of the joint instrument for 2002 Aug. 27 at 09:30 UT, with baselines up to $12000 \lambda$ :

1. The $N R H$ produces the central regular pattern (see Fig. 1b): a densely covered parallellogram with some linear

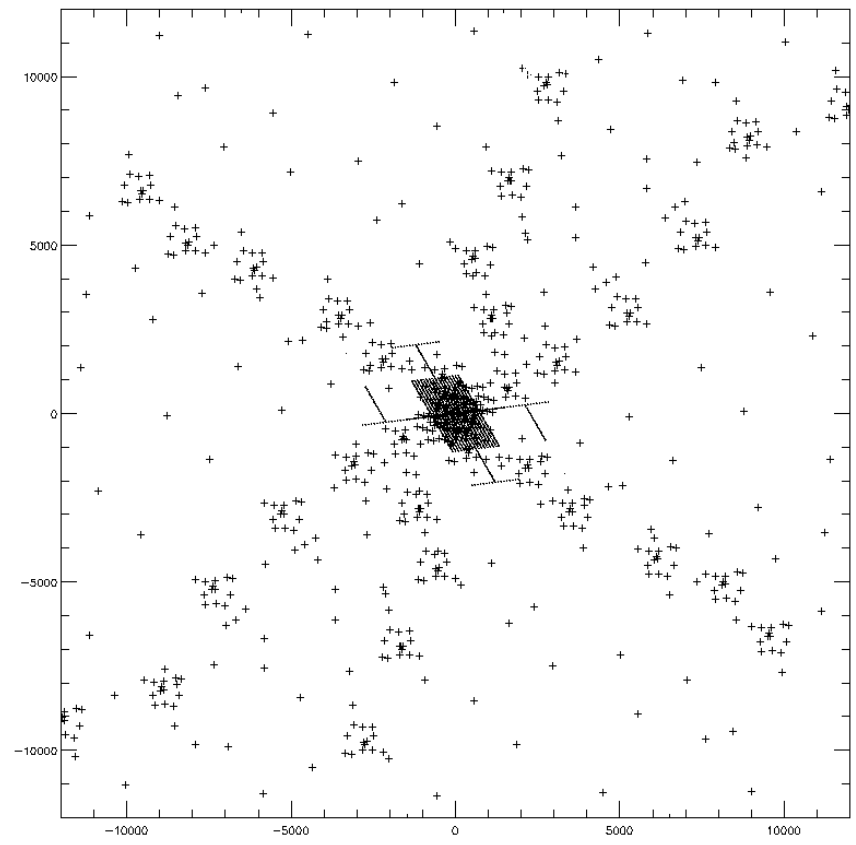

(a)

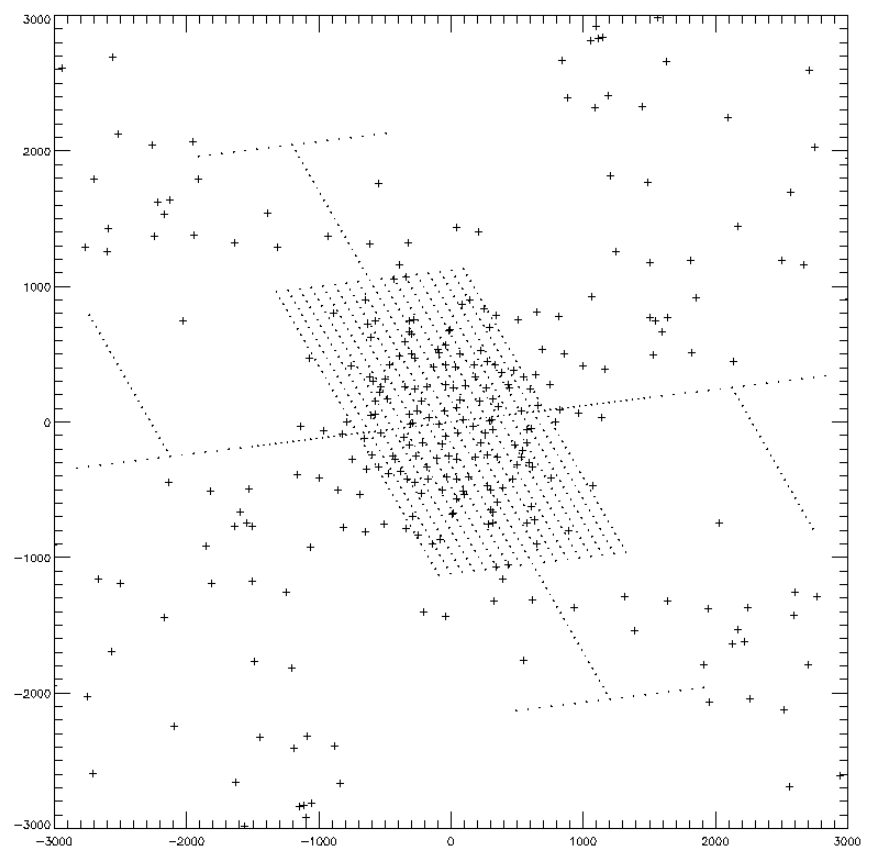

(b)

Fig. 1. Actual $u v$ coverage $\left(\mathrm{rad}^{-1}\right)$ for 2002 Aug. 27, 09:30 UT with $N R H+G M R T$. a) Coverage with baselines up to $12000 \lambda$; b) zoom-in of the central area showing the overlap between $N R H$ and GMRT coverages. GMRT data are marked by + , while $N R H$ data are marked by dots.

extensions, limited to values of $r_{u v}=\sqrt{u^{2}+v^{2}}$ smaller than $\approx 3000 \lambda$. This results from the regular antenna distribution of the $N R H$. 
2. The GMRT produces an irregular and much more extended pattern, consisting of:

- a central set of points corresponding to baselines from the central compact array of GMRT, which has a substantial overlap with the parallellogram of the $N R H$. We refer to the area of overlap between the inner baselines of the GMRT and NRH as $A_{\text {overlap; }}$;

- similar patterns with fewer points, distributed over 6 irregular arms, which correspond to baselines between the central array and the arm antennas;

- isolated points corresponding to baselines between the arm antennas.

The composite dirty lobe $L_{\mathrm{d}}$ is the FT of this $u v$ coverage and is the sum of $N R H$ and $G M R T$ dirty lobes $L_{\mathrm{d} R H}$ and $L_{\mathrm{d} G M R T}$. Due to the regular $N R H u v$ coverage $L_{\mathrm{d} R H}$ comprises of a pencil beam with classical "sinc" side lobes repeated over a period $\approx 1^{\circ}$. (larger than the size of the sun) on both axes (Fig. 2a).

Within one period, most of the flux of $L_{\mathrm{d} R H}$ is concentrated in a small area surrounding the main pencil beam, with extent $\approx 2$ or 3 times the width of the pencil. The resulting dirty image of the sun looks like a smoothed version of the sun, with side lobes $<20 \%$ near compact sources.

The situation is somewhat different for $L_{\mathrm{d} G M R T}$. The GMRT $u v$ coverage is much more sparse and irregular and the beam is very complex (see Fig. 2b): the narrow main pencil beam contains only a small fraction of the flux and is sourrounded by a complicated pattern of large side lobes. At larger distances from the main pencil beam, the structure of lobe is aperiodic (no regular grating lobes as for the $N R H$ ) and exhibits features of various scales and amplitudes, the most intense ones being at distances somewhat smaller than the size of the sun. As a result, the GMRT alone:

- cannot give a reliable snapshot image of the whole sun, especially of the large scale features;

- the dirty image of several sources on the sun is complex and difficult to understand visually, and requires a cleaning procedure.

Similarly, the benefits of merging GMRT data with $N R H$ data is not clear from a visual inspection of the dirty composite images (which appear visually to be similar to NRH dirty images plus some "small scale noise"). The benefits are obvious only from the composite clean images.

\section{Data selection and processing}

We present observations of the sun at $327 \mathrm{MHz}$ on Aug. 27, 2002 between 9 and 10 UT in this paper. We chose this day because there are complex sources on the sun, which present a challenge for snapshot imaging. The GMRT observations were integrated for a duration of $17 \mathrm{~s}$, while the $N R H$ data were integrated for a duration of $0.125 \mathrm{~s}$.

\subsection{Pre-processing}

The GMRT data were separately pre-processed in the following manner, using the standard AIPS software: a suitably interference free channel was first identified from the 128 channels

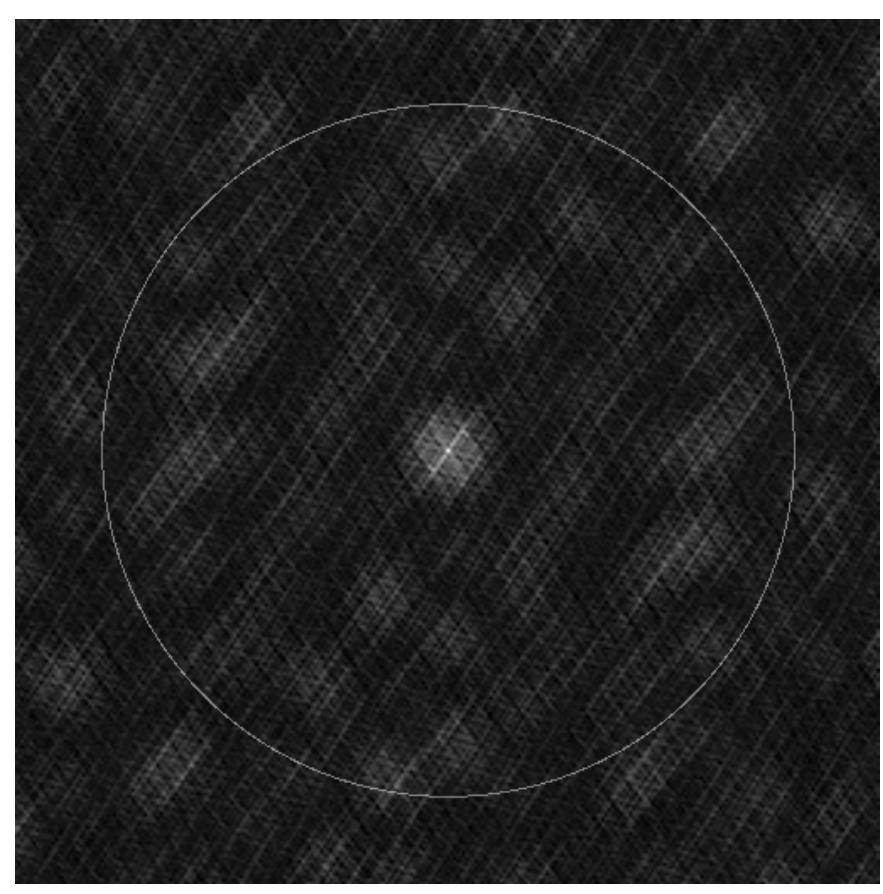

(b)

Fig. 2. Theoretical lobes for a) $N R H$ and b) $G M R T$. The circle represents the solar optical limb.

spanning $16 \mathrm{MHz}$ around $327 \mathrm{MHz}$. The data were then rigorously edited for bad antennas and baselines. The visibilities on the sun were then calibrated using those from the nearby phase calibrator $1021+219$. Before any subsequent processing, the $N R H$ data were therefore integrated over the $17 \mathrm{~s}$ intervals 
defined by the GMRT data. The NRH and GMRT visibilities were then intercalibrated in the following manner.

\subsubsection{Amplitude intercalibration}

The visibilty amplitudes from NRH and GMRT should be coherent, since both instruments use cosmic calibrators. NRH and GMRT $u v$ coverages overlap particularly densely on the area $A_{\text {overlap }}$ around the origin of the $u v$ plane (corresponding to baselines $<1 \mathrm{~km}$ ). Hence the sets of samples of the visibility on this area from both instruments should have comparable amplitude and merge homogeneously. This is especially true for the sun, for it dominates the system temperature for both the individual instruments. We noted that the amplitudes of the $G M R T$ visibilities were larger (as compared to the $N R H$ ones) by roughly one order of magnitude. This is primarily because the GMRT antennas are not fitted with solar noise calibrators, and the so-called " $T_{\text {sys }}$ " correction (Bastian 1989) can therefore not be applied. In other words, we cannot be certain about the absolute values of amplitudes of the GMRT visibilities (when observing the sun). We applied a multiplicative factor $a_{\text {intercal }}$ to the GMRT visibilities. The value of $a_{\text {intercal }}$ was determined in such a way that the mean value for the modulus of visibility, when averaged over $A_{\text {overlap }}$, was the same for both sets of data. We estimate that the accuracy of the resulting intercalibration is a few $\%$. Visual inspection of amplitude versus $u v$ distance plots for the composite data convinced us that this simple procedure gives satisfactory results.

\subsubsection{Position intercalibration}

The absolute positions of sources as observed by the two instruments can differ for several reasons:

1. For merging the visibilities, data are extracted from the standard processing algorithms of each instrument. Since fringe stopping for solar observations is complex and not necessarily done in one step, this can produce slowly varying position differences between $N R H$ and GMRT data at this stage.

2. The inhomogeneities due to gravity waves in ionospheric electron density can produce apparent shifts of the order of $1^{\prime}$ at $327 \mathrm{MHz}$, varying on time scales $\sim 30 \mathrm{~min}$ (see for instance Mercier 1996, and references therein). These effects are of local origin and would be totally uncorrelated between NRH and GMRT sites.

It is obvious that the position intercalibration must be better than the expected resolution. This was achieved in the following manner: for each $17 \mathrm{~s}$ interval, 2D dirty images were separately calcultated for $N R H$ and GMRT through a fast Fourier Transform (fft), including only baselines in the range common to both instruments (baselines $<2 \mathrm{~km}$, which makes this range somewhat larger than $A_{\text {overlap }}$ ), in order to achieve comparable resolutions. We then defined the translation required to make the maximum of GMRT dirty image coincide with the maximum of $N R H$ dirty image. This enables us to calulate the phase shifts that must be applied to all GMRT baselines in order to make the spatial origin identical for both instruments. We find that these differences in position were $\approx 0.15 R_{\mathrm{S}}$ and vary by less than $0.02 R_{\mathrm{S}}$ for the duration of our observations (9-10 UT, Aug. 27, 2002).

\subsection{Processing}

The processing comprises essentially of two steps: getting a "dirty" image and cleaning it, in order to make it visually understandable. When a single intense compact source is present, we can also estimate its dimensions by fitting a 2-D Gaussian directly to the observed visibilities. As we will see below, this allows us to measure sizes smaller than what would have been possible via inspection of clean composite images.

\subsubsection{Dirty image}

The dirty image is merely the Fourier Transform $(F T)$ of the joint set of visibilities. The fft algorithm has the advantage of being efficient, but it requires that spatial frequencies corresponding to the various baselines are distributed on a regular square grid in the $u v$ plane. This is not the case because $N R H$ and GMRT baselines are not simply commensurable (GMRT antennas are randomly distributed). This situation is usual in radioastronomy and regridding is commonly used. It consists of replacing the observed samples of the visibility by samples interpolated on a regular grid. We show hereafter that it is not convenient for snapshot images of a complex and wide object such as the sun with NRH and GMRT.

According to Shannon's theorem, if $w$ is the total angular width of the sun, its visibilty must be sampled with a step $u_{\text {sample }}$ which satisfies:

$u_{\text {sample }}<\frac{1}{w}$

Taking $w \sim 45^{\prime}=0.013 \mathrm{rad}$ at $327 \mathrm{MHz}, u_{\text {sample }}=77 \mathrm{rad}^{-1}$. The corresponding distance between neighboring antennas (projected normal to the line of sight) is $\lambda \times u_{\text {sample }}=69 \mathrm{~m}$. The sampling condition (1) is fullfilled for NRH baselines (at least far from local noon for observations before January 2003) but not for the GMRT baselines, especially for large baselines involving the distant arm antennas (which yield the enhanced spatial resolution). Thus interpolated values of the visibility for large baselines can be strongly biased and produce spurious effects such as "ghosts" of the actual sources. We emphasize that the condition (1) should be strictly satisfied for interpolating the visibility, since there can be several sources on the sun that are separated by more than $2 R_{\mathrm{S}}$.

In order to overcome this difficulty, we have defined a grid in the $u v$ plane with a period $u_{\text {grid }} \ll u_{\text {sample. In this way the }}$ observed visibilities can be ascribed to the nearest node of the grid, with only a small error. In other words, we keep the exact values measured for the visibility and make a small error ("round-up" error) $<0.5 u_{\text {grid }}$ on the spatial frequencies, which is much smaller than the minimum possible scale of variation of the visibility in the $u v$ plane, according to Eq. (1). In this way, we avoid the problem of interpolation in the $u v$ plane, at the expense of handling large arrays in fft computations. 
In our data processing we used arrays of dimension $1536 \times$ 1536 and restricted the longest baseline to $17000 \lambda$ (beyond this value GMRT uv coverage is too sparse for snapshots, see Sect. 3.2.2 below). Hence $u_{\text {grid }} \approx 22 \mathrm{rad}^{-1}$ and the round-up error in spatial frequencies is $<11 \mathrm{rad}^{-1}$, which is $\ll u_{\text {sample }}$. Since $N R H$ and GMRT taken together usually give fewer than 1000 visibility samples, most of the elements of the $1536 \times 1536$ array in the $u v$ plane are zero. When computing the image through a fft, the resulting field is $1 / u_{\text {grid }} \approx 8 \mathrm{deg}$. This field is much larger than the maximum width of the sun observable with the $N R H$ (see Eq. (1)), and we therefore retain only its central part.

\subsubsection{Cleaning procedure}

We first briefly recall the basic idea of CLEAN deconvolution. When the $u v$ coverage is sparse and irregular, the dirty lobe $L_{\mathrm{d}}$ has a complicated shape, with large side lobes at distances from the main peak that are much larger than its half power beam width $(H P B W)$. The contribution of a point on the object is not localized within one $H P B W$ of $L_{\mathrm{d}}$ on the dirty image, and this makes the dirty image difficult to interpret visually. CLEAN replaces the convolution of each point with the dirty lobe by a convolution with a clean lobe $L_{\mathrm{c}}$ (which is usually a Gaussian, with no sidelobes). The amplitude and $H P B W$ of the clean lobe are similar to that of the dirty lobe. The procedure is iterative, starting with the brightest points on the dirty image. The clean image thus depends "locally" (i.e., within one $H P B W$ ) on the object and is thus visually more understandable. For technical reasons the replacement is only partial at each step (see for instance Thompson et al. 1986, Chap. 11). In the $u v$ domain, the CLEAN process can be viewed as one where the holes in the $u v$ coverage are filled up via interpolation. However it is well known that CLEAN works rather poorly for continuous objects that have a wide spectrum of spatial scales. Some remedies have been proposed to alleviate this problem. In particular, when the object involves two well separated spatial scales (e.g., few compact sources superposed on the quiet sun), a two-scale CLEAN procedure can be used (Wakker \& Schwarz 1991). One can also try to subtract a model of the quiet sun from the dirty image. CLEAN then works on a dirty image which supposedly results only from some isolated compact sources. The model of quiet sun is added afterwards to the cleaned image for obtaining a final clean image.

The cleaning procedure we developed here is a multi-scale version of CLEAN (MS - CLEAN) adapted to objects with features of various scales (Delouis 1998). The basic idea is to first produce several filtered dirty images $I_{\mathrm{d} 0}, \ldots, I_{\mathrm{d} n}$ from the original dirty image $I$. Each filtered dirty image involves a relatively narrow range of spatial scales. For this purpose, we use successive filters $F_{0}, \ldots, F_{n}$ in the $u v$ plane, each filter selecting progressively smaller spatial scales:

- $F_{0}$ is a low-pass filter with cut-off $u_{\mathrm{e} 0} \approx 3-4 u_{\text {sample }}$ (recall that $u_{\text {sample }} \approx 3.5 u_{\text {grid }}$ and that the observable field of view is $\left.1 / u_{\text {sample }}\right)$.

- The filters $F_{1}, \ldots, F_{n}$ filters are band-pass filters with internal radii $u_{\mathrm{i} 1}=u_{\mathrm{e} 0}, \ldots, u_{\mathrm{in}}=a^{n-1} u_{\mathrm{e} 0}$ and external radii $u_{\mathrm{e} 1}=a u_{\mathrm{i} 1}=a u_{\mathrm{e} 0}, \ldots, u_{\mathrm{en}}=a^{n} u_{1}$, where $a \approx 3$ in order to limit the spatial scale ranges.

The edges of these filters are smooth and their sum is unity.

The first filtered dirty image $I_{\mathrm{d} 0}$ is very smooth and comprises of only a few independent pixels. It can be cleaned using smooth dirty and clean beams $L_{\mathrm{d} 0}$ and $L_{\mathrm{c} 0}$ deduced from $L_{\mathrm{d}}$ and $L_{\mathrm{c}}$ through a filtering with $F_{0}$. Thus the largest structures in the dirty image can be described in terms of only a small number of independent smooth components. The problem of describing a large scale structure with a large number of narrow compoments (if the CLEAN algorithm were directly applied) is then reduced to that of describing a source in components $\approx 4$ times narrower than the widest source that can be described. It is obvious that the number of iterations (and with it, the possibility of producing artefacts) in the CLEAN procedure will be drastically reduced.

The same argument can be applied to other filtered dirty images: each case is not very different from the case of a relatively small number of compact sources ("compact" relative to the dirty lobe). The final clean image is simply the sum of the filtered clean images.

After some trials we found that the best results were obtained under the following conditions:

- The GMRT uv coverage gets increasingly sparse for progressively longer baselines. At $327 \mathrm{MHz}$ there are only isolated points for baselines $>17000 \lambda$. This could lead to artefacts when cleaning dirty images from complex objects. We found that the best compromise between reducing these artefacts and preserving the spatial resolution could be achieved by taking a clean lobe $L_{\mathrm{c}}$ for which the width of its $F T$ is $\approx 0.35 \times u v_{\max }=0.35 \times b_{\max } / \lambda$, where $b_{\max }$ is the longest baseline under consideration. In simulations we could take $u v_{\max }=17000$ or 12000 , whereas for real data we had to take $u v_{\max }=12000$ only, probably because of imperfect calibration. In other words, $M S-C L E A N$ works poorly for sparse $u v$ coverages. The resulting $H P B W$ of the clean lobe was $\approx 1^{\prime}$.

- We took a maximum index $n=3$ for the filters (i.e., a central zone and 3 concentric rings). This allows us to cover a spatial frequency range up to $\approx 10^{4} \operatorname{rad}^{-1}$ with a ratio $a \approx 3$ for the ratio between the external and internal radii of filters.

\subsubsection{Estimating the width of an intense compact source}

The resolution corresponding to the width of our final clean beam is much lower than what could be expected from the $G M R T$ size. As explained earlier, this limitation arises because we try to obtain images from a complex object with a coverage that becomes very sparse for long baselines. However, for particularly simple objects, (e.g., when the composite image contains a single intense, compact source) it is possible to estimate the source size by fitting a Gaussian model directly to the amplitude of the visibility over the whole $u v$ range observed by the GMRT. It is then possible to compare this derived size with what is directly measured on clean images. Of course, this 
method is meaningful only when there is a dominant compact source, and cannot be applied when several sources with similar intensities co-exist.

\section{Results}

We first checked the expected performance of our method using simulated brightness distributions that are similar to the actual distribution on the day of our joint observations. We found that this procedure gives us a reliable method of anticipating the limitations when we process actual data. We show here the results of two representative simulations.

\subsection{Simulations}

\subsubsection{The models for the radio sun}

We defined a class of solar models which contain several features commonly observed at $327 \mathrm{MHz}$. They comprise structures of various sizes, ranging from the size of the quiet sun, down to $0.05 R_{\mathrm{S}}=52^{\prime \prime}$. These structures are derived from a simple mathematical model. The brightness distribution in the $1 \mathrm{D}$ analog of this model can be written as

$b(x)=\left(1-\left(\frac{x}{a}\right)^{e_{1}}\right)^{e_{2}}, \quad-a<x<a$,

$b(x)=0, \quad|x|>a$.

The parameters a, $e_{1}$ and $e_{2}$ define the half-power width of the structure, the flatness of its maximum and the slopes of its sides, respectively. In practice, we took a 2D model with an elliptical section, where the tilt of the ellipse major axis is also a free parameter. The model of the sun is the sum of $\approx 30$ such structures with various positions, sizes, flatness, tilt and amplitudes. The structures are:

- a relatively flat structure for the quiet sun, with total extent $42 \times 38^{\prime}(\mathrm{EW} \times \mathrm{NS})$, and $T_{B}=10^{6} \mathrm{~K}$.

- several "thermal" regions with various slopes, widths in the range $6-9$ ', various tilts and excess brightness of some $10^{5} \mathrm{~K}$ above the quiet sun.

- two coronal holes, one very elongated, with steep sides and depression depths $\approx 3 \times 10^{5} \mathrm{~K}$,

- a number of compact elliptical "non thermal" sources with sizes 1-3', various tilts and excess brightness of some $10^{5} \mathrm{~K}-3 \times 10^{7} \mathrm{~K}$ above the quiet sun,

- a faint CME-like arch whose brightness decreases with height and with distance from the central axis.

Using these ingredients, we produced two particular models for our simulations:

1. a relatively simple model (model 1 ) in which only 4 "non thermal" sources are intense ( $T$ up to $3 \times 10^{7} \mathrm{~K}$ ) and therefore dominate the other structures;

2. a more complex model (model 2), which has "non thermal" sources with $T \sim 10^{5} \mathrm{~K}-5 \times 10^{6} \mathrm{~K}$, so that they do not dominate the other features as in model 1.

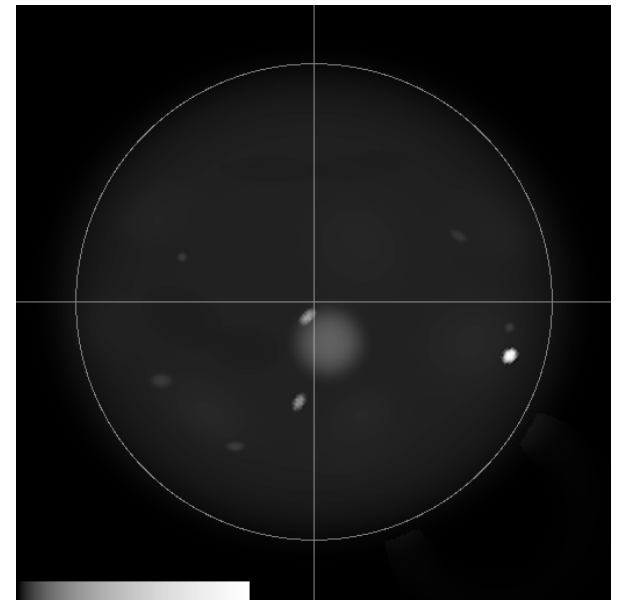

Fig. 3. Model 1 adopted for the simulations.

\subsubsection{Model 1}

Figure 3 shows model 1 . It is similar to several instances encountered in the real data of Aug. 27, 2002. It consists of:

- a bright western source with $T_{B}=3 \times 10^{7} \mathrm{~K}$;

- two relatively dimmer sources near the disk center and extended emission (of size $4^{\prime}$ ) between these two central sources.

The gray scale for Fig. 3 (and the subsequent figures) was chosen so as to optimize its visual appearance on paper prints. The intensity scale is indicated on the lower left corner: it is linear for low levels and progressively saturates at higher levels. This highlights low level features (CLEAN artefacts, in particular).

All other features (including the quiet sun) are hardly visible. Figure 4 shows the clean images obtained by the $N R H$ alone (a), GMRT alone (b) and $N R H+G M R T$ (c) when using the actual $u v$ coverages provided by the NRH and GMRT at 09:30 UT on Aug. 27, 2002. This means that the GMRT baselines which were flagged for the actual data of Aug. 27, 2002 were not used here either. The synthesized clean beam is shown at the bottom left corner of each figure. For cases b and c, it has an extent of 49". Even for this relatively simple model, the improvement in quality of the composite clean image (c) is quite evident: the resolution is much better than the $N R H$-only image, and there are fewer spurious large scale structures than in the $G M R T$-only image.

\subsubsection{Model 2}

Model 2 is shown in Fig. 5. It differs from model 1 in that the the nonthermal sources are dimmer, which allows us to discern the other features better: the quiet sun, patches of "thermal" emission, coronal holes, faint compact sources and a CME-like arch in the SW.

Figure 6 shows the clean images obtained by the $N R H$ alone (a), GMRT alone (b) and NRH + GMRT (c), using the same $u v$ coverage as for model 1:

The $N R H$ image is smooth (faint compact sources are not seen), but large scale structures are reliable, although they 


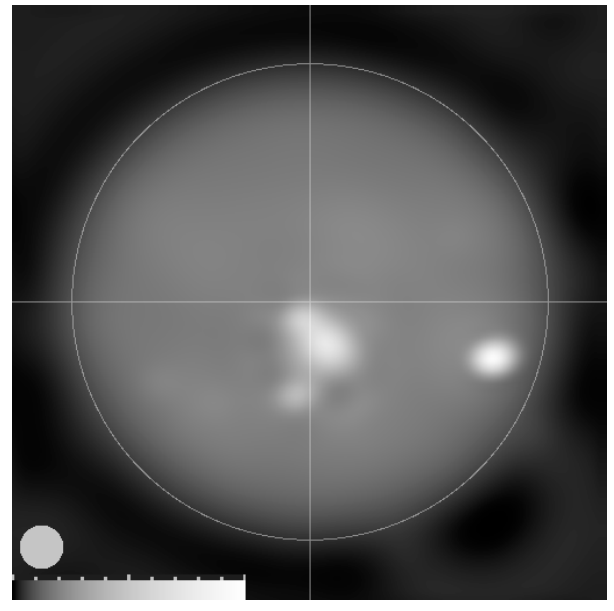

(a)

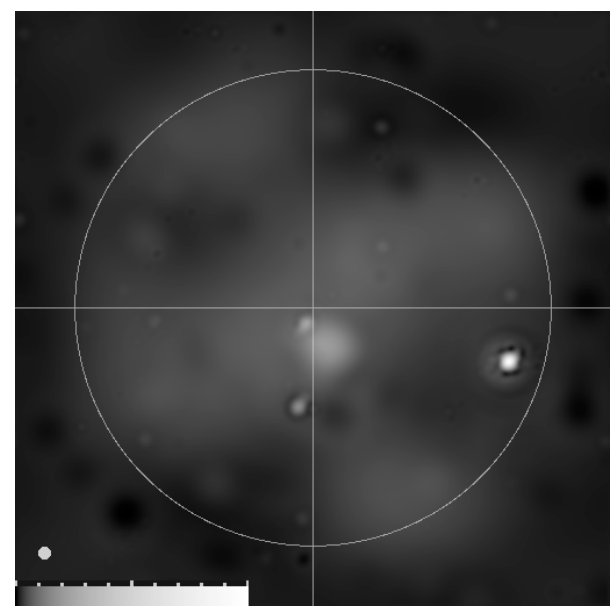

(b)

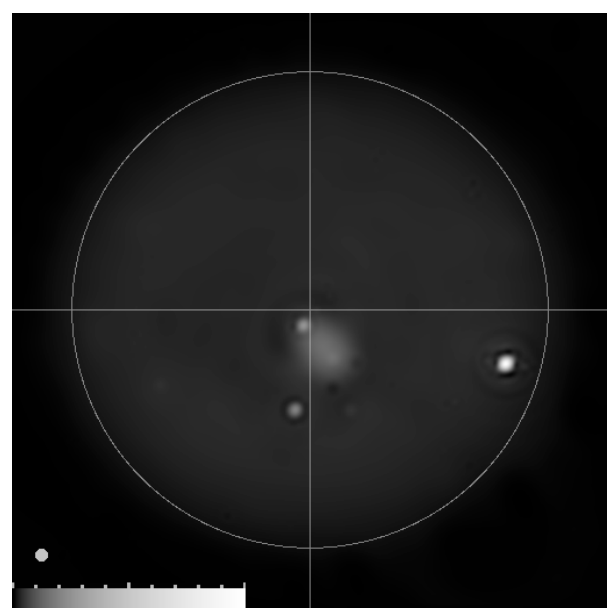

(c)

Fig. 4. Clean image for model 1: a) $N R H$ alone, b) GMRT alone, c) $N R H+G M R T$. The circle indicates the optical solar limb. The size of the clean beam is indicated in the lower left hand corner.

appear with a reduced contrast. The faint CME beyond the south west limb is satisfactorily evident. Conversely, due to

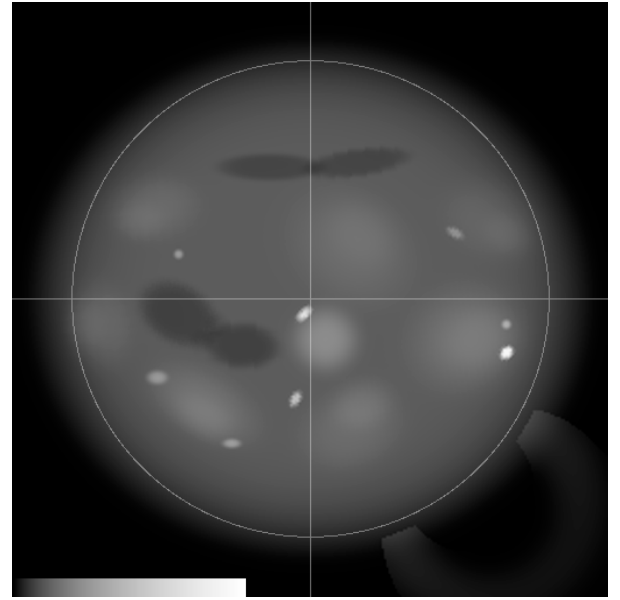

(a)

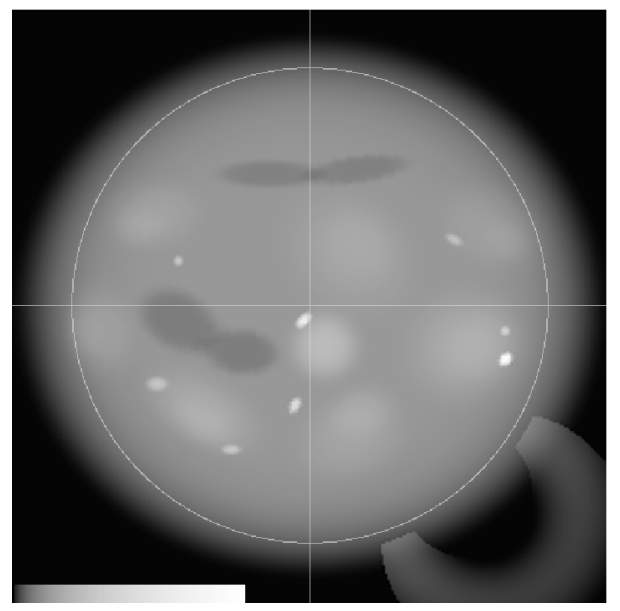

(b)

Fig. 5. a) and b): model 2 adopted for the simulations: a more complex sun. Panel a) shows an overview of the model. The gray scale has been chosen to enhance lower levels in panel b).

the greater complexity of the object, the clean image from $G M R T$ alone is practically unusable: large scale structures cannot be recognized, and only the two most intense compact sources can be identified. The improvement in quality of the composite image (c) is also evident, although limitations due to the sparse $u v$ coverage more apparent than for model 1 :

- the edges of coronal holes are blurred;

- low level artefacts (both positive and negative) appear around coronal holes and thermal regions;

- some of the faintest compact sources are difficult to distinguish from artefacts.

The CME is less visible in Fig. 6c than in Fig. 6a, but this is only due to the higher contrast of Fig. $6 c$, and because of its superior resolution.

Figure 7 shows the composite clean image obtained with the $u v$ coverage that could be provided if all GMRT antennas were functioning. It is somewhat better than Fig. 6c: faint 


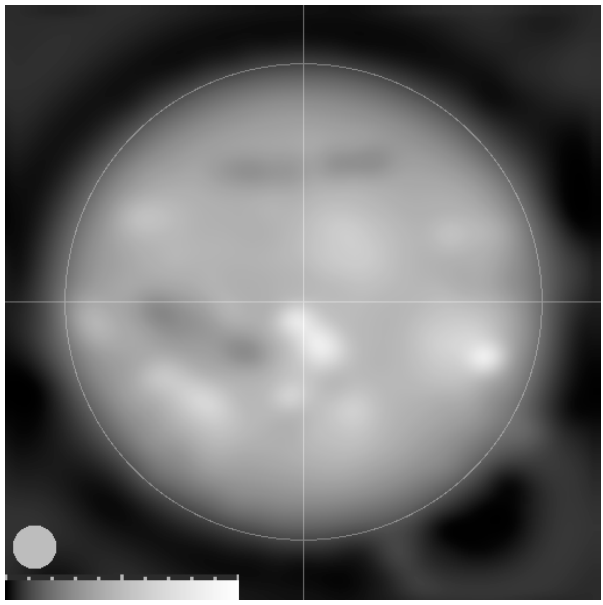

(a)

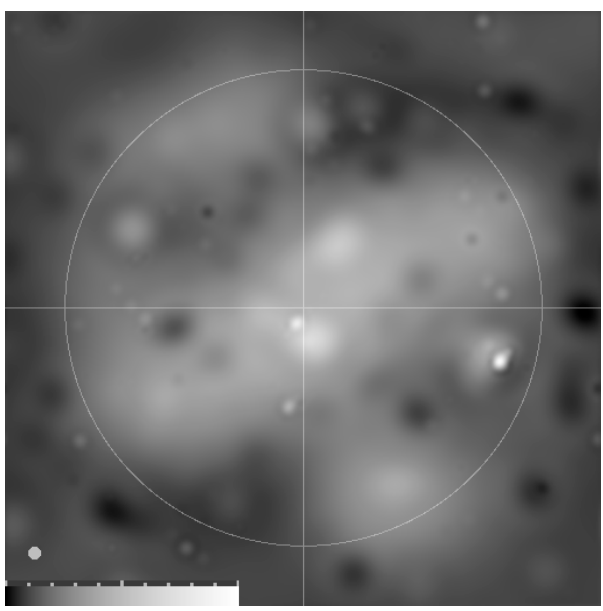

(b)

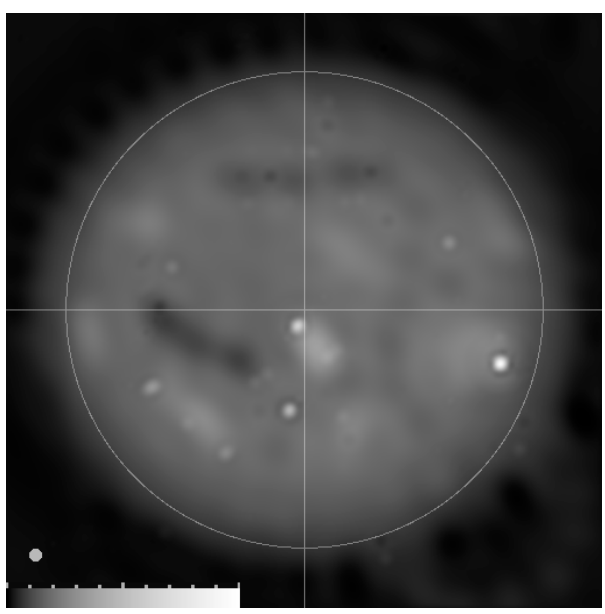

(c)

Fig. 6. Clean image for model 2: a) $N R H$ alone, b) $G M R T$ alone, c) $N R H+G M R T$.

compact sources are more reliable and the northern coronal hole has fewer artefacts.

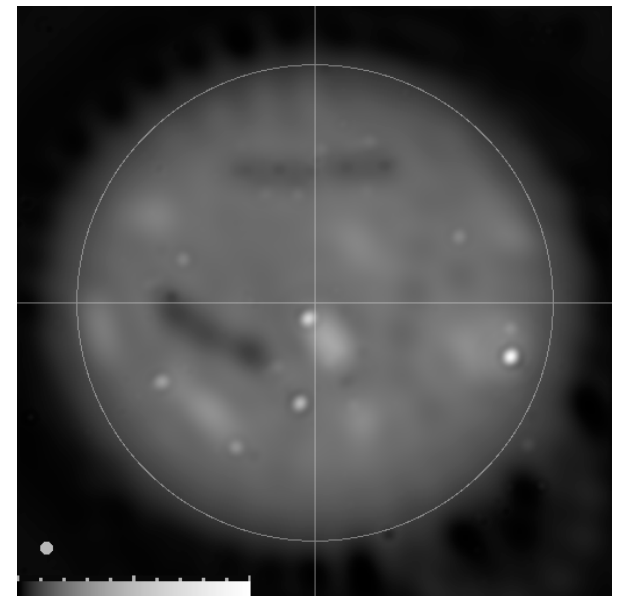

Fig. 7. Clean composite $(N R H+G M R T)$ image for model 2, using all the 435 possible GMRT baselines.

As expected, the benefits of merging $N R H$ and GMRT data are obvious when compact sources are present. However, it may be noted that low contrast extended features (e.g., the coronal holes of model 2) are also better imaged with the composite instrument. Model 2 illustrates the limits of what can be hoped from composite snapshots of a complex object with sparse extended $u v$ coverage.

In the preceding simulated examples, we had introduced no calibration errors (i.e., deviations in gains and phases). We have also produced images including random phase deviations in the simulated visibilities as a simple means of approximating the effect of phase calibration errors. We found that calibration errors are the principal cause of clean artefacts on the final images, and can effectively limit the resolution: for rms phase deviations $>20 \mathrm{deg}$, clean artefacts become noticeable and the extent of the accepted $u v$ coverage needs to be reduced.

\subsection{Observed data}

We now turn our attention to the data actually observed between 09:02:23 UT and 09:57:54 UT on Aug. 27, 2002. During this time, there was a strong ongoing noise storm near the west limb (0.69 $R_{\mathrm{S}}$ west, $0.34 R_{\mathrm{S}}$ south), hereafter referred to as " $W$ ". There were also two weaker noise storm sources near the disk center $\left(0.13 R_{\mathrm{S}}\right.$ east, $0.12 R_{\mathrm{S}}$ south and $0.13 R_{\mathrm{S}}$ east, $0.34 R_{\mathrm{S}}$ south), referred to as "N" and "S" respectively. While the western noise storm source remains compact throughout the duration of our combined observations, the two sources near disk center evolve significantly, showing marked extensions at certain times. We have selected three $17 \mathrm{~s}$ snapshots, which highlight some of these features and illustrate the capability of our combined imaging technique.

Figure 8 shows $17 \mathrm{~s}$ snapshots centered around 09:20:25 UT, using data from $N R H$ alone (a), GMRT alone (b) and from $N R H+G M R T$ (c). The $u v$ coverage has already been shown in Fig. 1: the relatively sparse, extended $u v$ coverage 


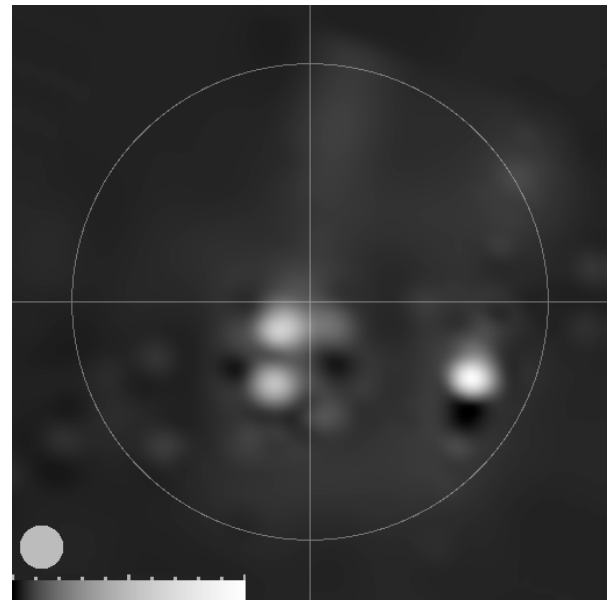

(a)

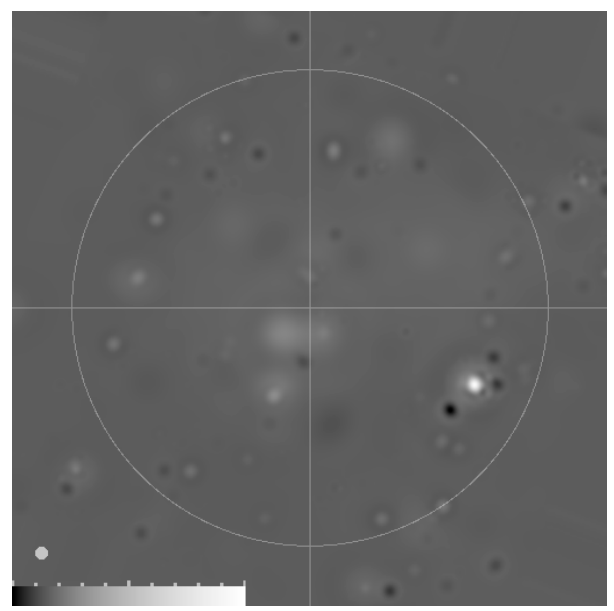

(b)

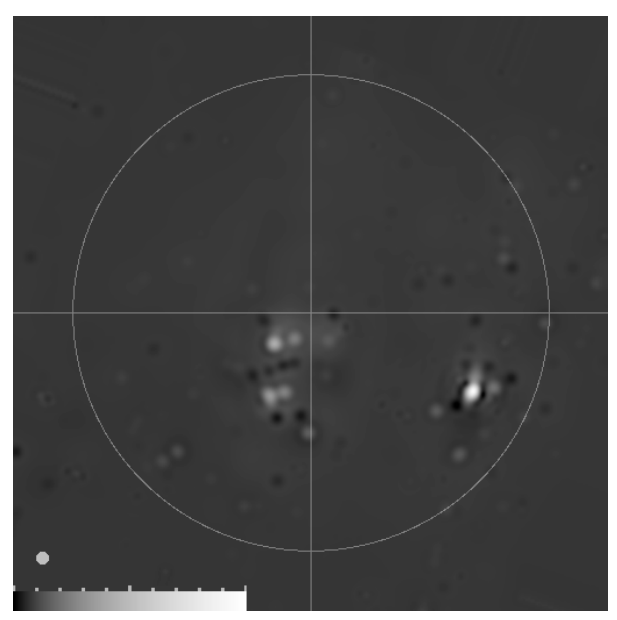

(c)

Fig. 8. Clean image at 09:20:25 UT from a) $N R H$ alone, b) GMRT alone, c) $N R H+G M R T$.

of the GMRT, which is complemented by the dense, short baseline $u v$ coverage of the $N R H$.
The improvement in resolution (when the GMRT data are used) is evident: the half-power size of the clean beam in Figs. $8 \mathrm{~b}$ and $8 \mathrm{c}$ (lower left hand corner) is $49^{\prime \prime}$. The rms dynamic range for the composite image (Fig. 8c) is 244, while the max dynamic range is 11 . On the GMRT-only image (Fig. 8b), we note that:

- artefacts are spread over the whole field. The relatively strong level of negative artefacts produces a grey background for the picture;

- the two central sources are not properly imaged. This is especially true of " $N$ " which is smooth in Fig. $8 \mathrm{~b}$, whereas it is more compact on the composite image (Fig. 8c).

The composite image shows artefacts which are both fainter (as is evident from the darker background) and more localized to the vicinity of sources. This must be compared to simulations with model 1 (which is very similar to this actual case), for which GMRT alone gives satisfactory results for compact sources and a much lower level for artefacts (Fig. 4b). Since the data processing is the same for the simulations and for real data, we think that the difference could be ascribed to imperfect phase calibration of GMRT data, and possibly to phase shifts arising from the $30 \mathrm{~dB}$ solar attenuators, which are switched on for solar observations and off for calibrator observations.

The western source " $\mathrm{W}$ " is brightest at 09:13:39. Figure 9 shows clean snapshot images at this time from $N R H$ alone (a), $G M R T$ alone (b) and from NRH + GMRT (c). Clearly, the improved resolution is provided by the GMRT baselines, while $N R H$ data helps in reducing clean artefacts. Note that, as for the preceding case, "N" is also poorly imaged by the GMRT alone. The resolution of the composite image, as in earlier cases, is $49^{\prime \prime}$. The rms dynamic range of this image is 370 and the max dynamic range is 21 .

The source "W" in this snapshot is the smallest structure present in our observations. It is barely resolved with the clean beam size of $49^{\prime \prime}$. However, since there is only one intense source present at this time, we can use the step described in Sect. 3.2.3 (fitting a Gaussian to the visibilities) to estimate a size of $\approx 1^{\prime}$. Using simulations, we checked that this derived size is not appreciably affected by the faint sources " $N$ " and " $\mathrm{S}$ " and by the faint halo surrounding " $\mathrm{N}$ ". High time resolution $N R H$ data show that the sources " $\mathrm{N}$ " and " $\mathrm{S}$ " (and "W", to a lesser extent) exhibit continual small-scale motions. Since the time resolution of composite images is limited by the $17 \mathrm{~s}$ integration time of the GMRT data, it is possible that this snapshot image has "smeared over" such small scale motions. It follows that the source size of 1 ' we derive for " $\mathrm{W}$ " is likely an overestimate of its actual size.

Figure 10 shows 17 s snapshots centered around 09:04:04 on Aug. 27, 2002, from NRH alone (a), GMRT alone (b) and from $N R H+G M R T$ (c). It illustrates the ability of the composite instrument to resolve and image extended emission reliably. The extended emission of around 200" that lies in between, and to the west "N" and " $\mathrm{S}$ " is imaged much better in the composite image than in any of the individual images. None of the instruments can give a satisfactory image of "N": the resolution of $N R H$ is too low, and GMRT, in spite of its high resolution, 


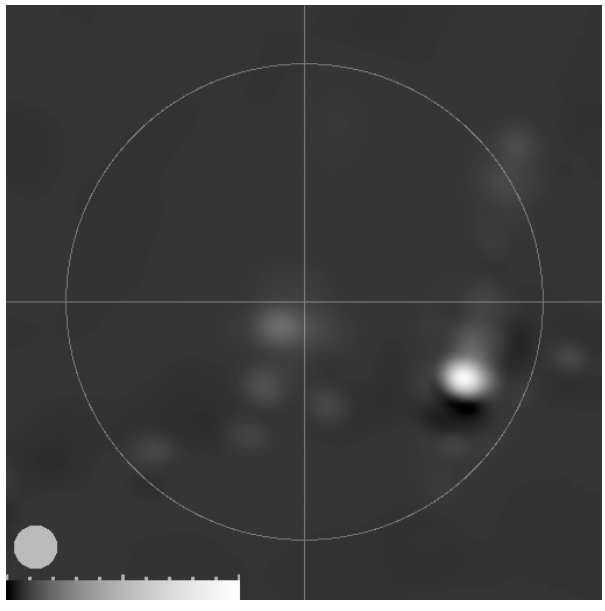

(a)

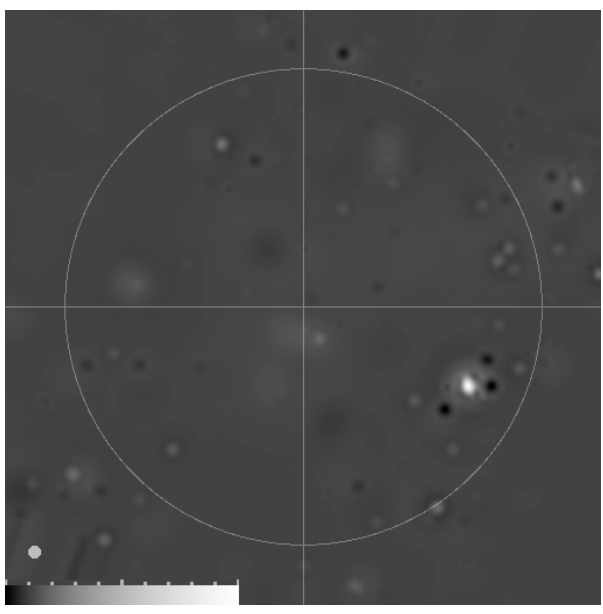

(b)

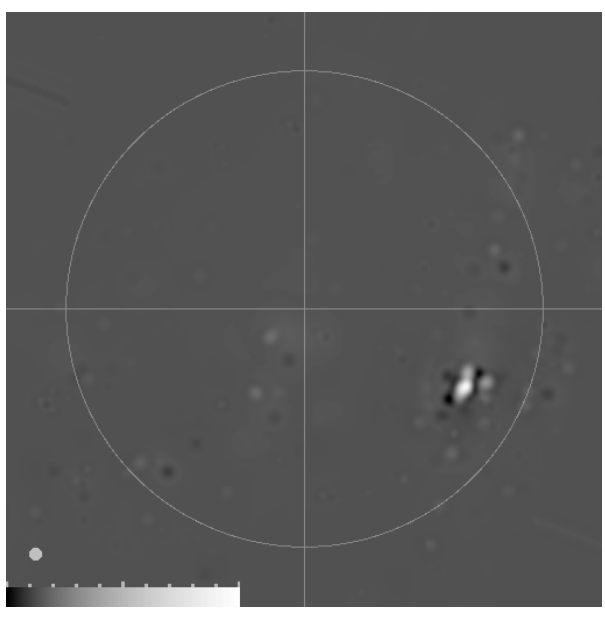

(c)

Fig. 9. Clean image at 09:13:39 UT from a) $N R H$ alone, b) $G M R T$ alone, c) $N R H+G M R T$. Resolutions are the same as for Fig. 8.

does not image the bright core of "N" properly. The resolution of this composite image is $49^{\prime \prime}$ as for the other images.

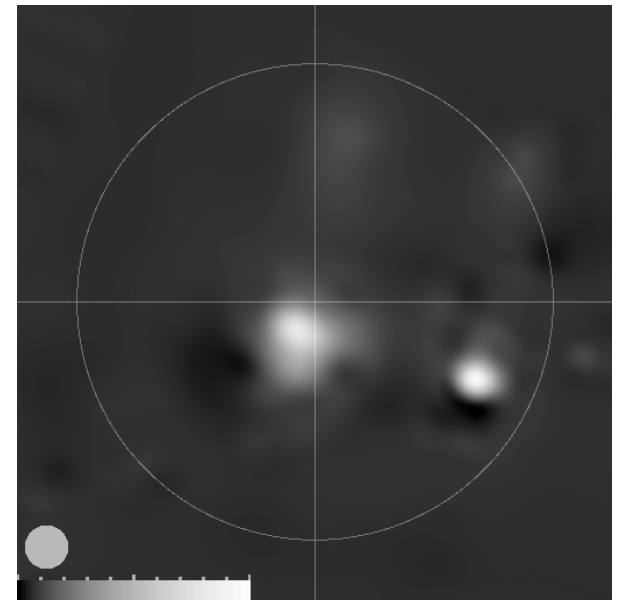

(a)

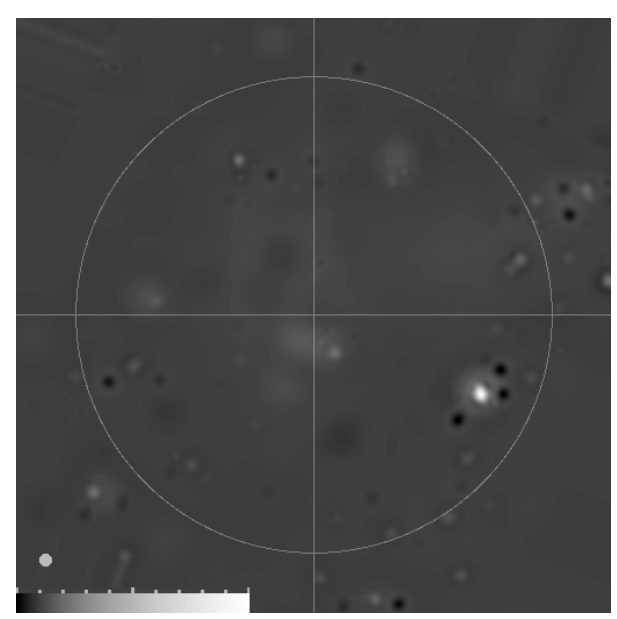

(b)

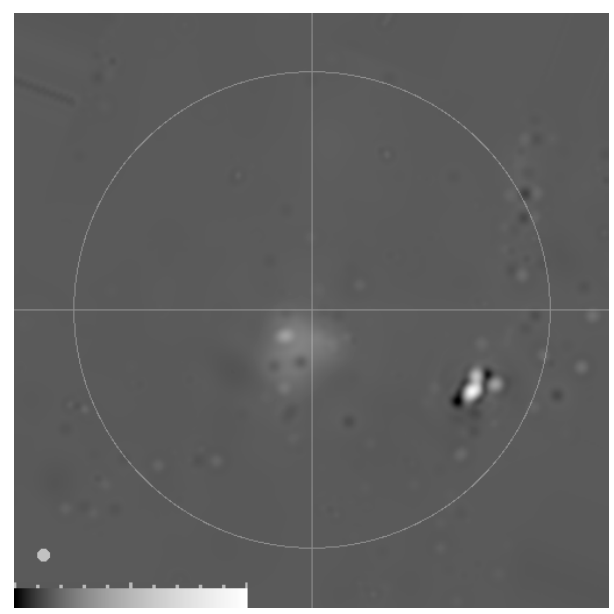

(c)

Fig. 10. Clean image at 09:04:04 UT from a) $N R H$ alone, b) GMRT alone, c) $N R H+G M R T$. Resolutions are the same as for Fig. 8 .

The rms dynamic range of this image is 280 , and the max dynamic range is 14 . 
Upon an examination of high time resolution $N R H$ data, it is evident that the relatively faint halo near " $\mathrm{N}$ " on the composite images is in fact a group of short, bright and almost unpolarized type III bursts, beside the noise storms "S" and "N". In addition to the short time scale motions of " $\mathrm{S}$ " and "N" mentioned earlier, this shows that the observations presented here are only illustrative of the possibilities of the joint instrument. Higher time resolution observations with this joint instrument are very much needed in order to derive physical interpretations of solar radio emissions in this frequency range.

\section{Summary and conclusion}

We have demonstrated the capablities of a new $N R H+G M R T$ composite instrument for obtaining snapshot images of the sun at $327 \mathrm{MHz}$. We have shown examples from simulations with two models (of differing complexity) of the radio sun, and from real observations of complex, evolving noise storm sources on Aug. 27, 2002.

The simulations show the capabibility of the joint instrument, to produce snapshot images of a complex sun, with structure sizes ranging the resolution $\left(\approx 50^{\prime \prime}\right)$ up to the size of the whole sun $\left(\approx 40^{\prime}\right)$. The main conclusions from the simulations are:

- GMRT and NRH are highly complementary: long $G M R T$ baselines provide resolution, whereas dense $N R H$ $u v$ coverage at short baselines prevent aliasing of large structures which can occur with GMRT alone.

- The composite instrument allows a substantial advance in snapshot imaging of complex objects. The resolution and dynamic ranges of composite images are far better than those of images from the individual instruments (Fig. 7).

- The quality of the calibration can limit the resolution and produce appreciable artefacts.

We produced composite $17 \mathrm{~s}$ snapshot images (from actual observations of the sun on Aug. 27, 2002) of structures between $60^{\prime \prime}$ and $\sim 200^{\prime \prime}$ in size with a resolution of $49^{\prime \prime}$ and rms dynamic ranges of 250-420. The quality of the composite image is far better than those of images from the individual instruments (e.g. Fig. 8). The max dynamic ranges of the snapshot maps are $\gtrsim 20$. To the best of our knowledge, these are the highest dynamic range snapshot maps of the sun at meter wavelengths. Until now, high dynamic range radio maps of the sun were typically made by synthesis imaging over time periods of a few hours. As mentioned in the introduction, high dynamic range images would be essential in studying phenomena like bright radio bursts occurring along with (fainter phenomena like) coronal mass ejections.

These observational results are only illustrative of the capabilities of the technique we have employed. Merging GMRT and $N R H$ data will potentially yield $\approx 1000$ baselines per snapshot and would yield very high resolution, high dynamic range maps. This is roughly equivalent to simultaneously using the capabilities of the VLA B, C and D arrays (the comparison concerns only the number of baselines, since these VLA configurations cannot be used simultaneously to produce snapshots).
The resolution of the maps presented in this paper was limited to $49^{\prime \prime}$, which is well below what one would crudely expect from the GMRT size. This limitation in the resolution is mostly because:

- We are dealing with snapshot images and the $u v$ coverage of the GMRT is sparse, especially for large baselines. The deconvolution procedure does not work well for complex objects under these conditions. We need to introduce substantial tapering of the visibilities (see Sect. 3.2.2) in order to limit artefacts. This is a basic limitation on snapshot images, which would not concern synthesis composite images because the $u v$ coverage (for synthesis images) would be much less sparse, especially for large baselines. When the object is simple enough (e.g. Fig. 9), however, one can achieve (from snapshot data) the full resolution corresponding to the size of GMRT by using classical model fitting.

- We had to flag several GMRT baselines owing to malfunctioning antennas and radio frequency interferences.

- We encountered some calibration problems with GMRT data, which could arise from the unknown phase errors arising from the $30 \mathrm{db}$ attenuators that need to be inserted when the GMRT antennas point at the sun.We have checked the effect of calibration errors with simulations, and it is clear that they make a significant difference in the level of CLEAN artefacts in the final images. In order to reduce them to an acceptable level, we had to increase the above-mentioned tapering. The final resolution of $49^{\prime \prime}$ is thus the best compromise between achieving the best possible resolution and maintaining a reasonably low level of clean artefacts.

For future observations, it is desirable to:

- achieve the best possible calibration, especially for the $G M R T$. The GMRT is probably more sensitive than $N R H$ to phase deviations because of its sparser $u v$ coverage;

- use the shortest possible integration time for GMRT observations $(\approx 2 \mathrm{~s}$, rather than $17 \mathrm{~s}$ as in the current observations). This is because radio bursts can vary on timescales much shorter than $17 \mathrm{~s}$, and the data presented here could have integrated over several potentially interesting timevarying phenomena (as was the case for the snapshot at 09:04:04, Aug. 27, 2002).

This could lead to images with better resolution and dynamic ranges. We could then conclusively answer some long-standing questions in solar physics, as mentioned in the introduction.

Acknowledgements. We thank the staff of the GMRT and the NRH that made these observations possible. The GMRT is run by the National Centre for Radio Astrophysics of the Tata Institute of Fundamental Research, India. The $N R H$ is supported by the Observatoire de Paris, CNRS and Région Centre. We warmly acknowledge continued financial support from the French embassy in India without which this work could not have been accomplished. We thank also an anonymous referee for clarifying remarks. 


\section{References}

Ananthakrishnan, S., \& Pramesh Rao, A. 2002, Giant Metrewave Radio Telescope, in Proc. Int. Conf. on Multicolour Universe, ed. R. K. Manchanda, \& B. Paul, TIFR, Mumbai, 233 http://www.gmrt.ncra.tifr.res.in/gmrt_hpage/Users/ doc/doc.html

Bastian, T. S. 1989, Solar Imaging with a Synthesis Telescope, in Synthesis Imaging in Radio Astronomy, ed. R. A. Perley, F. R. Schwab, \& A. H. Bridle, ASP Conf. Ser., 6

Bastian, T. S. 1994, ApJ, 426, 774

Delouis, J.-M. 1998, Thesis dissertation

Gopalswamy, N., White, S. M., \& Kundu, M. R. 1991, ApJ, 379, 366

Habbal, S. R., Ellman, N. E., \& Gonzalez, R. 1989, ApJ, 342, 594

Habbal, S. R., Mossman, A., Gonzalez, R., \& Esser, R. 1996, JGR, 101,19943

Kerdraon, A. 1979, A\&A, 71, 266

Kerdraon, A., \& Delouis, J.-M. 1997, in Coronal Physics from Radio and Space Observations; Proceedings of the CESRA workshop held in Nouan le Fuzelier, ed. G. Trottet (Springer), 192
Mercier, C. 1996, Ann. Geophysicae, 14, 42

Swarup, G., Ananthakrishnan, S., Kapahi, V. K., et al. 1991, Current Sci., 60, 95

Thompson, A. R., Moran, J. M., \& Swenson, G. W. 1986, Interferometry and Synthesis in Radio Astronomy (New York: J. Wiley and sons)

Wakker, B. P., \& Schwarz, U. J. 1991, Radio Interferometry: Theory, techniques and Applications, ed. T. J. Cornwell, \& R. A. Perley, IAU Coll., 131, ASP Conf. Ser., 19

White, S. M., Kundu, M. R., \& Gopalswamy, N. 1992, ApJS, 78, 599

Willson, R. F., Kile, J. N., \& Rothberg, B. 1997, Sol. Phys., 170, 299

Willson, R. F., Redfield, S. L., Lang, K. R., Thompson, B. J., \& St. Cyr, O. C. 1998, ApJ, 504, L117

Willson, R. F. 2000, Sol. Phys., 197, 399

Zlobec, P., Messerotti, M., Dulk, G. A., \& Kucera, T. 1992, Sol. Phys., 141,165 